\title{
Clinicopathologic characteristics and management of minute esophageal lesions diagnosed by narrow-band imaging endoscopy
}

Authors

Institutions
Takashi Kumamoto $^{1}$, Kazuhiro Sentani ${ }^{2}$, Shiro Oka ${ }^{3}$, Shinji Tanaka ${ }^{3}$, Wataru Yasui ${ }^{2}$

Kumamoto Gastrointestinal Clinic, Hiroshima, Japan

2 Department of Molecular Pathology, Hiroshima University Institute of Biomedical and Health Sciences, Hiroshima, Japan

3 Department of Endoscopy, Hiroshima University Hospital, Hiroshima, Japan submitted

21. December 2015 accepted after revision 13. June 2016

\section{Bibliography}

DOI http://dx.doi.org/

10.1055/s-0042-110788

Published online: 30.8.2016

Endoscopy International Open

2016; 04: E927-E932

(c) Georg Thieme Verlag KG

Stuttgart - New York

E-ISSN 2196-9736

\section{Corresponding author}

\section{Takashi Kumamoto}

Kumamoto Gastrointestinal Clinic

1-5-2 Otemachi

Naka-ku

Hiroshima 730-0051

Japan

Phone: +81-82-544-0606

Fax: +81-82-544-0377

kumamoto-clinic@guitar.ocn. ne.jp
Background and study aims: Magnifying narrowband imaging (NBI) endoscopy enables the diagnosis of minute esophageal neoplasia. We aimed to evaluate clinicopathological diagnosis of minute esophageal neoplasia by using magnifying NBI endoscopy and biopsy.

Patients and methods: In total, 309 patients (127 men and 182 women) with minute esophageal lesions of intrapapillary capillary loop (IPCL) type IV were enrolled. Of these patients, 249 underwent biopsy for histologic diagnosis and also for treatment. Of the 249 patients, 123 underwent follow-up with endoscopy. We analyzed the clinicopathologic characteristics and prognosis of these lesions after biopsy.

Results: Of the 249 biopsied lesions, we histologically diagnosed 11 as high-grade intraepithelial neoplasia (HGIN), 41 as low-grade intraepithelial

\section{Introduction}

\section{$\nabla$}

Most patients with advanced esophageal cancer have poor prognosis. Therefore, early diagnosis is particularly important for esophageal cancer. Magnifying narrow-band imaging (NBI) has been reported to be useful for the diagnosis of early esophageal [1 -5] and pharyngeal cancer [6-10]. Furthermore, magnifying NBI enabled the diagnosis of minute esophageal and pharyngeal lesions with a diameter of approximately $1 \mathrm{~mm}$. However, reports on the diagnosis of minute esophageal lesions by magnifying NBI are scarce [11]. In a previous report, we revealed the efficiency of NBI and biopsy in the diagnosis of minute pharyngeal lesions [12]. In the current report, we describe the extent of efficacy of NBI in the diagnosis of minute esophageal lesions.

When a brownish area is detected in the esophagus on unmagnified NBI, magnifying NBI is performed to aid classification of the intrapapillary capillary loop (IPCL), which involves microvessels of esophageal and pharyngeal squamous epithe- neoplasia (LGIN), and 197 as non-neoplasia (Non-N) including inflammation. Six of the 11 HGINs and 11 of the 41 LGINs showed slight elevation. Background coloration was observed in 9 of 11 HGINs, 34 of 41 LGINs, and 33 of 197 NonNs. Of the 249 biopsied lesions, 147 were microscopically measurable. The average diameter was $1.4 \mathrm{~mm}$ for HGINs and $0.8 \mathrm{~mm}$ for LGINs. Of the 123 patients who underwent post-biopsy followup, 93 (76\%) showed no lesions at the biopsied sites during the NBI examinations and were suspected to have undergone complete resection by biopsy.

Conclusions: Biopsy was useful for diagnosis and treatment of minute esophageal lesions, diagnosed as IPCL type IV by magnifying NBI endoscopy.

lial lesions [13-15]. Moreover, when IPCL type IV or V is observed, neoplasia is suspected.

No consensus exists regarding management of minute esophageal lesions. However, our first option for treatment of this condition is biopsy to obtain a histologic diagnosis, with the aim of complete resection. No previous reports have described use of biopsy for minute esophageal lesions. In the current report, we describe clinicopathologic diagnosis and follow-up findings after biopsy for minute esophageal neoplasia.

\section{Patients and Methods \\ $\nabla$ \\ Patients}

In principle, all patients aged $>30$ years who require gastrointestinal endoscopy at our clinic are examined using magnifying NBI. From September 2008 to December 2011, a total of 309 consecutive patients (127 men and 182 women) with minute esophageal lesions, as determined to be IPCL type IV by magnifying NBI, were enrolled in 
this study ( $\square$ Table 1 ). Minute esophageal lesion was defined to be smaller than the width of closed biopsy forceps, that is, approximately $2 \mathrm{~mm}$. During this period, we routinely performed 7158 upper gastrointestinal tract endoscopic examinations by magnifying NBI endoscopy (in 3286 men and 3872 women). The drinking and smoking habits of 264 patients (122 men and 142 women) were assessed. Of the 309 lesions, 249 were biopsied, whereas the others were followed up. The non-biopsied lesions included difficult lesions with any risk of post-biopsy bleeding, such as lesions on a large vein or lesions in patients taking anticoagulants. Detailed histologic assessments were performed for the 249 biopsied lesions; of them, 123 were followed up with endoscopy until December 2013.

We examined clinicopathologic characteristics of patients, endoscopic findings of the lesions, and prognosis after biopsy.

\section{Instruments}

A magnifying endoscope that was capable of $\times 80$ magnification (GIF H260Z; Olympus Co. Ltd, Tokyo, Japan), a standard videoendoscopy system (EVIS LUCERA; Olympus), and a NBI system (Olympus) were used in this study.

\section{Endoscopic examination}

All endoscopic examinations were performed by the first author (TK). A magnification hood (MB-46, Olympus) was attached to the tip of the endoscope. Intravenous access and pulse oximetry monitoring were established prior to the examination. Most of the examinations were performed under intravenous sedation with pethidine hydrochloride $(17.5 \mathrm{mg}-70 \mathrm{mg}$ ) and midazolam ( $0.5 \mathrm{mg}-4 \mathrm{mg}$ ). The esophagus is not as sensitive to endoscopy as is the pharynx. Because deep sedation may cause respiratory depression, sedation should be carefully performed. The pharynx and esophagus were primarily observed using NBI from the beginning of the examination. The iodine dye method of the esophagus was also used in high-risk cases of esophageal cancer, that is, male drinkers and smokers older than age 50 at the final stage of endoscopy. The stomach and duodenum were mainly observed using white light. Magnifying NBI was used for all esophageal lesions with noticeable brownish areas during NBI, and IPCL classification was performed. The brownish areas were set to the 12 o'clock position by rotating the endoscope and were then magnified. IPCL classification was performed following previously described criteria $[13,14]$. Background coloration of the magnifying NBI was also assessed. Almost all the minute lesions were observed with white light after the NBI observations. The 267 lesions were biopsied during the withdrawal stage to avoid the influence of post-biopsy bleeding on the examination of the stomach and duodenum. Biopsy of lesions adjacent to veins was avoided due to the risk of post-biopsy bleeding. Biopsies were performed with disposable biopsy forceps (FB-210K, Olympus) to obtain the largest and deepest biopsy specimen possible, to ensure complete resection. The lesions were set at the 6 o'clock position by rotating the endoscope, and were then biopsied under NBI. A precise lesion-centered biopsy was intended. For biopsy, the plane of the opened biopsy forceps should be horizontal, which is achieved by rotating the forceps handle. To avoid post-biopsy bleeding, all anticoagulants were discontinued from Day 3 before the biopsy to Day 3 after the biopsy, if possible. Patients who underwent biopsy were asked to remain in the clinic for 2 to 3 hours, including a 1-hour period for post-sedation recovery. Annual magnifying NBI sessions were recommended for all the biopsied patients, but only 123 of the 249 biopsied
Table 1 Patient characteristics $(n=309)$.

\begin{tabular}{|l|l|}
\hline Characteristics & No. \\
\hline Age, year (range) & \\
\hline Men & $61.4 \pm 11.6(35-83)$ \\
\hline Women & $60.9 \pm 11.3(32-84)$ \\
\hline Sex, men/women & $127 / 182$ \\
\hline Alcohol consumption & $264(122$ men, 142 women) \\
\hline Yes & $99(78$ men, 21 women $)$ \\
\hline No & 165 (44 men, 121 women) \\
\hline Smoker & $264(122$ men, 142 women) \\
\hline Yes & $97(83$ men, 14 women) \\
\hline No & 167 (39 men, 128 women) \\
\hline Esophageal cancer history & 3 \\
\hline Yes & 306 \\
\hline No & 13 (gastric cancer: 6 ; colon can- \\
\hline Other cancer history & cer: 4 ; breast cancer: 1 ; laryngeal \\
\hline Yes & cancer: 1 ; and thyroid cancer: 1$)$ \\
\hline
\end{tabular}

patients eventually underwent follow-up using magnifying NBI endoscopy. To reduce interobserver variation, results of the NBI and magnifying NBI examinations were independently evaluated by 2 endoscopists (TK and SO). When the evaluations differed, a consensus was reached by reviewing the magnifying NBI images.

Histologic methods and criteria for pathologic diagnosis The biopsy specimens were extended and fixed to a styrene foam plate by fine acupuncture needles [12]. The biopsy specimens were processed as described previously [12], and the pathologic diagnoses were independently evaluated by 2 pathologists (KS and WY). The diagnosis of neoplasia followed the criteria proposed by the World Health Organization [16]. In the current study, neoplasia was classified as low-grade intraepithelial neoplasia (LGIN) or high-grade intraepithelial neoplasia (HGIN). These criteria are based on architectural and cytologic atypia. Architectural atypia was determined by assessing the proliferative cell distribution and the tumor front, whereas cytologic atypia was assessed using cell size, nuclear arrangement, and nuclear size. Non-neoplasia lesions, such as inflammation, were classified as non-neoplasia (Non-N). The lesion diameters were measured at histology under light microscopy using the built-in measurement system of the light microscope, which enabled measurements accurate up to $0.1 \mathrm{~mm}$.

\section{Results \\ $\nabla$}

Of the 7158 patients (3286 men, 3872 women) who were examined at our clinic by magnifying NBI endoscopy from September 2008 to December 2011, 309 were diagnosed with minute esophageal IPCL type IV lesions. The clinicopathologic characteristics of the patients are shown in Table 1. Of the 309 patients, 127 were men and 182 were women. The overall frequency of the lesions was $4.3 \%$ in all the patients $(3.9 \%$ in men and $4.7 \%$ in women). Of the 264 patients (122 men, 142 women) in whom drinking and smoking habits could be assessed, 99 (78 men, 21 women) had a history of alcohol consumption and 97 (83 men, 14 women) had a history of smoking. Thus, a history of alcohol consumption was noted in $64 \%$ of men and $15 \%$ of women, 
whereas a history of smoking was noted in $68 \%$ of men and $10 \%$ of women. With regard to history of cancer, 6 patients had gastric cancer, 4 had colon cancer, and 3 had esophageal cancer.

All 309 lesions showed similar findings on both magnifying and unmagnified NBI examinations. $\odot$ Table 2 shows the endoscopic features of each histologic examination. IPCL type IV followed the previously described classification criteria $[13,14]$. As per the criteria, IPCL type IV lesions should fulfill 3 of the following 4 characteristics: dilatation, tortuous course, caliber change, and different shapes. Although almost all of the lesions were flat, 6 of 11 HGINs, 11 of 41 LGINs, and 29 of 197 Non-Ns were slightly elevated. Background coloration was observed on magnifying NBI [17,18] in 9 of 11 HGINs, 34 of 41 LGINs, and 33 of 197 Non-Ns ( $\bullet$ Table 2).

Of 309 lesions, 249 were biopsied to determine the histologic diagnosis and, if possible, achieve complete resection. The histologic diagnoses of the 249 biopsied lesions included HGINs in 11 cases, LGINs in 41 cases, and Non-Ns in 197 cases ( $\bullet$ Fig. $\mathbf{1}$ ). No cancerous lesions were observed. The study flow schema is shown in $\bullet$ Fig. 1. Moreover, $\bigcirc$ Fig. 2 shows a lesion diagnosed as HGIN. This HGIN lesion was slightly elevated, particularly when observed using white light ( $\boldsymbol{O}$ Fig.2a), and background coloration was also observed $(\boldsymbol{\bullet}$ Fig. $\mathbf{2 d}$ )

Of the 249 biopsied lesions, 147 could be measured. The distribution of the lesion diameters is shown in 0 Table 3 . The most frequently observed diameter was $0.5 \mathrm{~mm}$, and $82 \%$ of the lesions had a diameter $<1.0 \mathrm{~mm}$. The average lesion diameter was $0.65 \mathrm{~mm}$ for Non-Ins, $0.83 \mathrm{~mm}$ for LGINs, and $1.42 \mathrm{~mm}$ for HGINs; thus, HGINs were larger than LGINs. The smallest LGIN and HGIN were both $0.3 \mathrm{~mm}$ in diameter. The lower limit of LGIN diameters was $0.3 \mathrm{~mm}$, whereas the upper limit was $2.0 \mathrm{~mm}$; most LGINs had a diameter of $0.5 \mathrm{~mm}$ to $1.2 \mathrm{~mm}$. Cases of Non-Ns showed a similar distribution. In contrast, the lower limit of HGIN diameters was $0.3 \mathrm{~mm}$, whereas the upper limit was $3.0 \mathrm{~mm}$; there was no specific frequently observed diameter. In addition, $60 \%$ of lesions with a diameter of $>1.1 \mathrm{~mm}$ was either LGIN or HGIN, and all lesions with a diameter of $>2.1 \mathrm{~mm}$ were HGIN.

Of the 123 patients who underwent biopsy and received endoscopic follow-up, 93 (76\%) did not have any lesions at the biopsy site during NBI; thus, complete resection was observed as a result of biopsy in these patients. Of 30 patients with lesions remaining after biopsy, 24 patients received follow-up endoscopy and rebiopsy and 14 of 24 lesions disappeared after re-biopsying 1 to 4 times. The interval from first diagnosis to follow-up ranged from 6 months to 61 months (mean, 18.5 months).

The iodine dye method was also used in 678 patients (603 men, 75 women) of the 7158 patients, and we encountered 2 cases of HGINs with diameters $\geq 5 \mathrm{~mm}$, which were not detected during the prior NBI endoscopy on the same day.

\section{Discussion}

Magnifying NBI endoscopy is effective for diagnosing minute esophageal [19-21] and pharyngeal lesions [22-26]. Hence, in our institution, we routinely examine the pharynx and the esophagus with NBI at the start of the examination under careful sedation. We examined the IPCL type IV lesions not only with NBI but also under white light imaging, after diagnosis via magnifying NBI. The iodine dye method is also reportedly useful for diagnosing early esophageal cancers $[27,28]$, but magnifying NBI endoscopy is considered to be more specific, particularly for min-
Table 2 Endoscopic features of minute esophageal lesions according to histology.

\begin{tabular}{|llll|}
\hline & Non-N & LGIN & HGIN \\
\hline No. & 197 & 41 & 11 \\
\hline Sex, men/women & $76 / 121$ & $19 / 22$ & $5 / 6$ \\
\hline Location (U/M/L) & $31 / 130 / 36$ & $4 / 27 / 10$ & $0 / 8 / 3$ \\
\hline Macroscopic type (elevated/flat) & $29 / 168$ & $11 / 30$ & $6 / 5$ \\
\hline Background coloration (\%) & $33(17)$ & $34(80)$ & $9(82)$ \\
\hline
\end{tabular}

U: upper esophagus, M: middle esophagus, L: lower esophagus.

Non-N: non-intraepithelial neoplasia.

LGIN: low-grade intraepithelial neoplasia.

HGIN: high-grade intraepithelial neoplasia.

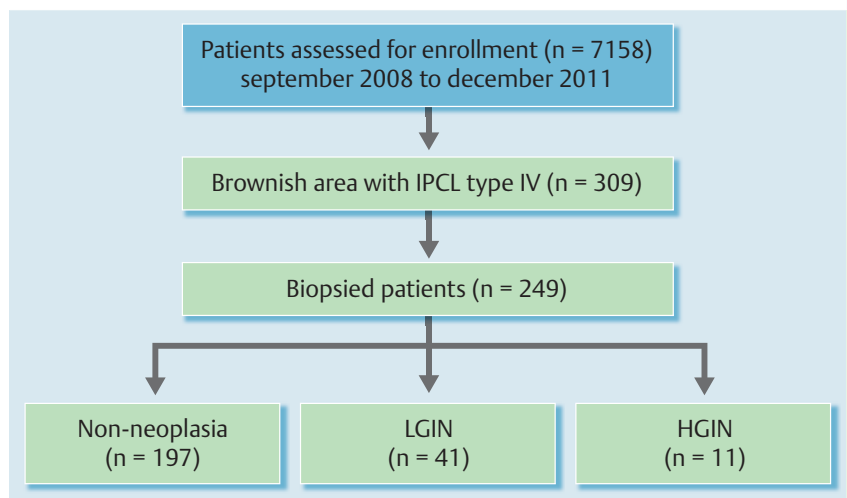

Fig. 1 Flow schema of the study.

ute esophageal lesions, as compared to the iodine dye method [29]. The iodine dye method is reported to pick up too many iodine-unstained minute esophageal lesions, including inflammatory changes [29], and if the iodine dye method is used after magnifying NBI endoscopy, a minute IPCL type IV lesion that is also unstained by the iodine dye method may be overlooked before biopsy due to the other multiple minute nonspecific iodineunstained lesions. Therefore, when a minute IPCL type IV lesion was diagnosed, we did not use the iodine dye method before biopsy. However, the iodine dye method is also known to be more sensitive than NBI for some cancer lesions and HGINs. In fact, we encountered 2 cases of HGIN with diameters $>5 \mathrm{~mm}$ that were not detected during the prior NBI endoscopy on the same day, but were diagnosed with the iodine dye method. Hence, we not only use NBI, but also the iodine dye method, particularly in high-risk cases.

The frequency of minute esophageal lesions was $4.3 \%$ overall (3.9\% in men and $4.7 \%$ in women). Because esophageal cancers occur predominantly in men with a history of alcohol consumption and smoking, we also expected the incidence of minute esophageal lesions to be higher among them. However, minute esophageal lesions were as prevalent among females as among males. Moreover, as many women as men had HGINs. Because more men than women had a history of alcohol consumption and smoking, those exposures may predispose an individual to carcinogenesis from HGINs. In the current study, 6 patients had gastric cancer and 4 had colon cancer. A history of other cancers may also be considered a risk factor.

The magnifying NBI endoscopic findings of the 309 lesions were similar among HGINs, LGINs and non-Ns. Moreover, the ultraminute HGINs and LGINs reportedly have comparable features under magnifying NBI endoscopy and in immunohistochemistry 


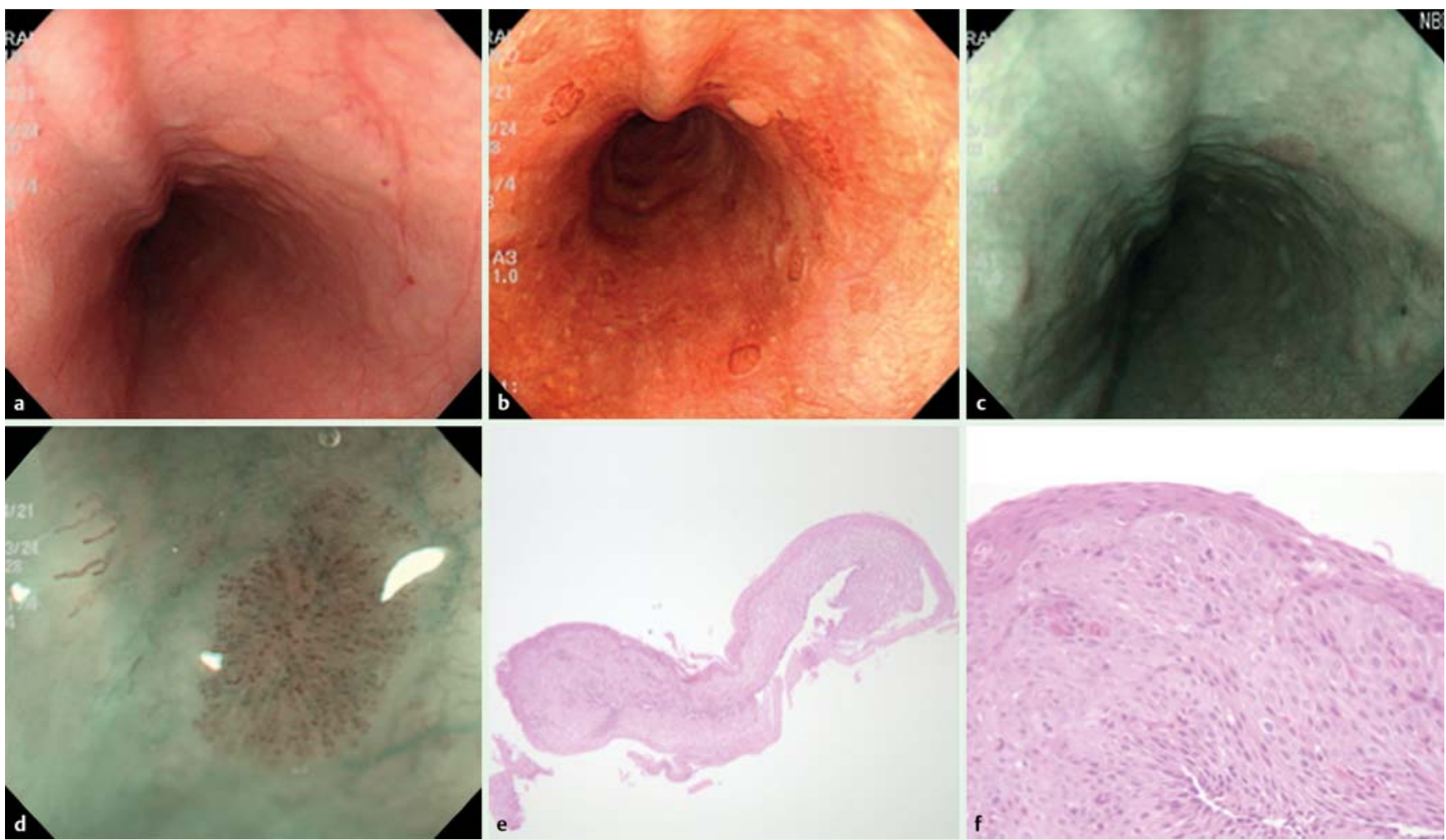

Fig. 2 Narrow-band imaging and histological images of a high-grade intraepithelial neoplasia. a The neoplastic lesion appears as slightly elevated reddish colored mucosa. b Endoscopic view of the lesion with iodine dye staining. The lesion is not stained by iodine dye. $\mathbf{c}$ Narrow-band imaging of panel A, showing a well-demarcated brownish area. $\mathbf{d}$ Magnified NBI view, showing an intrapapillary capillary loop type IV pattern. Irregular morphologic changes are observed in the superficial microvessels in the brownish area. Background coloration can be seen. e Low-power magnification image of the biopsied specimen, showing tumor front formation and complete epithelial layer invasion (hematoxylin and eosin [HE]; original, $\times 100$ ). $\mathbf{f}$ Histologically, the lesion shows abnormal cell size variation and increased nuclear size; it is accordingly diagnosed as a high-grade intraepithelial neoplasia [HE; original, $\times 400]$.

Table 3 Diameters of biopsied lesions.

\begin{tabular}{|lcccl|}
\hline Diameter $(\mathbf{m m})$ & No. & Non-N (\%) & LGIN (\%) & HGIN (\%) \\
\hline $0.1-0.2$ & 1 & $1(100)$ & $0(0)$ & $0(0)$ \\
\hline $0.3-0.4$ & 35 & $31(88)$ & $3(9)$ & $1(3)$ \\
\hline $0.5-0.6$ & 37 & $25(68)$ & $10(27)$ & $2(5)$ \\
\hline $0.7-0.8$ & 28 & $17(61)$ & $11(39)$ & $0(0)$ \\
\hline $0.9-1.0$ & 21 & $15(71)$ & $6(29)$ & $0(0)$ \\
\hline $1.1-1.2$ & 14 & $7(50)$ & $5(36)$ & $2(14)$ \\
\hline $1.3-1.4$ & 3 & $1(33)$ & $1(33)$ & $1(33)$ \\
\hline $1.5-1.6$ & 3 & $1(33)$ & $2(67)$ & $0(0)$ \\
\hline $1.7-1.8$ & 0 & $0(0)$ & $0(0)$ & $0(0)$ \\
\hline $1.9-2.0$ & 2 & $1(50)$ & $1(50)$ & $0(0)$ \\
\hline $2.1-2.5$ & 2 & $0(0)$ & $0(0)$ & $2(100)$ \\
\hline 3.0 & 1 & $0(0)$ & $0(0)$ & $1(100)$ \\
\hline
\end{tabular}

HGIN: high-grade intraepithelial neoplasia; LGIN: low-grade intraepithelial neoplasia; Non-N: non-intraepithelial neoplasia.

[11]. A slight elevation of the lesions was noted in $55 \%$ (6 of 11 ) of HGINs, $27 \%$ (11 of 41 ) of LGINs, and $15 \%$ (29 of 197) of Non-Ns, and may serve as a marker for HGINs. Background coloration is defined as a color change in the area between IPCLs [17]. Background coloration on magnifying NBI endoscopy, which is reportedly observed more frequently in HGINs than in LGINs $[17,18,30]$, was noted in approximately $82 \%$ of both HGINs (9 of 11 ) and LGINs (34 of 41) in our study, without any major difference. In addition, background coloration was only noted in $17 \%$ (33 of 197) of Non-Ns, a lower frequency than that for HGINs and LGINs. Hence that may serve as a marker for differentiating HGINS or LGINs from Non-Ns.

We used a disposable biopsy forceps to ensure the largest and deepest biopsy possible, for complete resection. The biopsied specimens were extended and fixed to a styrene foam plate. Because the extended specimens were small and had a diameter of $4 \mathrm{~mm}$ to $5 \mathrm{~mm}$, fine acupuncture needles were used for fixation. With these procedures, precise histologic diagnoses, including diameter measurement, were possible. The histologic diagnoses were HGIN in 11 cases, although no cancerous lesions were noted. Because HGINs are considered cancer precursors [31], minute esophageal lesions appear to be original lesions that can develop into cancers. The proportion of HGINs was lower than expected. Magnifying NBI endoscopy's low specificity for diagnosis of HGINs may be due to the fact that the minute esophageal lesions were at a very early stage in the growth of neoplasia and that histologic changes may be mild compared with larger lesions.

The lesion diameters were measured microscopically, up to an accuracy of $0.1 \mathrm{~mm}$, and a diameter survey was performed. At our clinic, we usually encounter typical outpatients with varied gastrointestinal symptoms, and follow-up is rare for cases of head and neck cancer. Therefore, the diameter distribution observed in the current study was considered close to the natural distribution. Moreover, the distribution appears to show the growth of the minute lesions. The average diameter of HGINs was larger than that of LGINs. The lower limit of LGIN diameters was $0.3 \mathrm{~mm}$, whereas the upper limit was $2.0 \mathrm{~mm}$; most LGINs had a diameter of $0.5 \mathrm{~mm}$ to $1.2 \mathrm{~mm}$. Non-Ns also showed a sim- 
ilar pattern in terms of lesion diameter. In contrast, the lower limit of HGIN diameters was $0.3 \mathrm{~mm}$, whereas the upper limit was $3.0 \mathrm{~mm}$; there was no specific frequently observed diameter. It appears that LGINs and Non-Ns are diameter-limited lesions, and some of them may be a source of HGINs. HGINs can grow horizontally over $2.0 \mathrm{~mm}$ and may lead to cancers.

Almost all lesions were flat. However, 6 of 11 HGINs and 11 out of 41 LGINs were slightly elevated. White light examination also tended to indicate a slight elevation. In addition to a horizontal lateral growth, some of the lesions may show vertical upward growth [32]. Minute esophageal lesions are considered to originate from the basal cell layer and grow horizontally and upward. Because HGINs occupy almost the entire epithelial mucosal layer, it may be possible for some of these lesions to show slight elevation.

Follow-up studies indicated that the resection rate was $76 \%$, which was similar to that for minute pharyngeal lesions in our previous report (79\%) [12]. Although annual endoscopic followup has not been completed in some cases, we believe that complete resections may be expected in some cases. We re-biopsied 24 incompletely resected lesions 1 to 4 times and 14 lesions achieved complete resection. Repeated biopsy seemed useful for incompletely resected lesions. We did not observe any rapidly growing lesions during endoscopic follow-up after biopsy.

Although HGIN lesions are considered to be cancer precursors, it is difficult to determine whether a lesion is HGIN with magnifying NBI endoscopy alone. Because the incidence of slight elevation was higher in HGINs than in LGINs, it may serve as a marker of HGINs, and thus, a slightly elevated minute lesion should be biopsied. Background coloration may not be useful as a means of differentiating between minute esophageal HGINs and LGINs. Because the average diameter of HGIN was larger than that of LGIN, lesion diameter may serve as a marker of HGIN. However, small HGINs of $0.3 \mathrm{~mm}$ and $0.6 \mathrm{~mm}$ in diameter were also present, hence lesion diameter may not be a definitive marker. Biopsy, therefore, appears to be necessary to confirm the diagnosis.

Endoscopic submucosal dissection or endoscopic mucosal resection has been performed for early mucosal esophageal cancers to ensure complete resection. However, it remains unclear whether we should follow up, biopsy, or endoscopically resect the minute lesions. We diagnosed 11 cases with HGINs by using biopsy, and complete resection was achieved in $76 \%$ of the biopsied lesions in this study. Hence, biopsy may not only be useful for diagnosis, but also for treatment of such minute lesions, and it may serve as first-line treatment.

The current study has several limitations. First, it is a single-center retrospective study with examinations performed by a single endoscopist. To reduce interobserver variation, the results were independently evaluated by 2 endoscopists. Second, in $19.4 \%$ of patients (60 out of 309 cases), lesions were mainly adjacent to veins and were not biopsied. Third, $50.6 \%$ of patients (126 out of 249 cases) were not followed up, although annual endoscopic follow up was recommended to all of the patients.

In conclusion, we diagnosed $4.3 \%$ of patients with minute esophageal lesions by using magnifying NBI endoscopy. Biopsy was considered useful for diagnosis and treatment of minute esophageal lesions, particularly those with positive background coloration, slight elevation, or diameters $>1 \mathrm{~mm}$.

\section{Competing interests: None}

\section{References}

1 Yoshida T, Inoue $H$, Usui $S$ et al. Narrow-band imaging system with magnifying endoscopy for superficial esophageal lesions. Gastrointest Endosc 2004; 59: 288-295

2 Muto M, Minashi K, Yano T et al. Early detection of superficial squamous cell carcinoma in the head and neck region and esophagus by narrow band imaging: a Multicenter randomized controlled trial. J Clin Oncol 2010; 28: 1566-1572

3 Muto M, Hironaka S, Nakane M et al. Association of multiple ligol-voiding lesions with synchronous and metachronous esophageal squamous cell carcinoma in patients with head and neck cancer. Gastrointest Endosc 2002; 56: 517-521

4 Muto M, Takahashi M, Ohtsu A et al. Risk of multiple squamous cell carcinomas both in the esophagus and the head and neck region. Carcinogenesis 2005; 26: $1008-1012$

5 Muto M, Katada C, Sano Yet al. Narrow band imaging: a new diagnostic approach to visualize angiogenesis in superficial neoplasia. Clin Gastroenterol Hepatol 2005; 3: 16-S20

6 Nonaka S, Saito $Y$. Endoscopic diagonosis of pharyngeal carcinoma by NBI. Endoscopy 2008; 40: 347-351

7 Muto M, Nakane M, Katada C et al. Squamous cell carcinoma in situ at oropharyngeal and hypopharyngeal mucosal sites. Cancer 2004; 101: $1375-1381$

8 Ugumori T, Muto M, Hayashi $R$ et al. Prospective study of early detection of pharyngeal superficial carcinoma with the narrowband imaging laryngoscope. Head Neck 2009; 31: 189-194

9 Watanabe A, Tsujie $H$, Taniguchi $M$ et al. Laryngoscopic detection of pharyngeal carcinoma in situ with narrowband imaging. Laryngoscope 2006; 116: 650-654

10 Matsuba H, Katada C, Masaki T et al. Diagnosis of the extent of advanced oropharyngeal and hypopharyngeal cancers by narrow band imaging with magnifying endoscopy. Laryngoscope 2011; 121: $753-$ 759

11 Goda K, Dobashi A, Yoshimura $N$ et al. Clinicopathological features of narrow-band imaging endoscopy and immunohistochemistry in ultraminute esophageal squamous neoplasms. Dis Esophagus 2014: 27, $267-275$

12 Kumamoto T, Sentani K, Oka S et al. Clinicopathological features of minute pharyngeal lesions diagnosed by narrow-band imaging endoscopy and biopsy. World J Gastroenterol 2012; 18: 6468 - 6474

13 Inoue $H$. Magnification endoscopy in the esophagus and stomach. Dig Endosc 2001; 13: 40-S41

14 Inoue H, Kaga M, Sato Yet al. Magnifying endoscopic diagnosis of tissue atypia and cancer invasion depth in the area of pharyngo-esophageal squamous epithelium by NBI enhanced magnification image: IPCL pattern classification. In: Cohen J (ed.) Advanced Digestive Endoscopy: Comprehensive Atlas of High Resolution Endoscopy and Narrowband Imaging. Oxford: Wiley-Blackwell; 2007: 49-66

15 Kumagai $Y$, Inoue $H$, Nagai $K$ et al. Magnifying endoscopy, stereoscopic microscopy, and the microvascular architecture of superficial esophageal carcinoma. Endoscopy 2002; 34: 369-375

16 Barnes L, Eveson JW, Reichart P et al. World Health Organization Classification of Tumours. Pathology and Genetics of Head and Neck Tumours. Lyon: IARC Press; 2005

17 Minami $\mathrm{H}$, Inoue $\mathrm{H}$, Ikeda $\mathrm{H}$ et al. Usefulness of background coloration in detection of esophago-pharyngeal lesions using NBI magnification. Gastroenterol Res Pract 2012; 2012: 529182

18 Minami $\mathrm{H}$, Isomoto $\mathrm{H}$, Inoue $\mathrm{H}$ et al. Significance of background coloration in endoscopic detection of early esophageal squamous cell carcinoma. Digestion 2014; 89: 6-11

19 Ishihara $R$, Inoue $T$, Uedo $N$ et al. Significance of each narrow-band imaging finding in diagnosing squamous mucosal high-grade neoplasia of the esophagus. J Gastroenterol Hepatol 2010; 25: 1410-1415

20 Ishihara R, Takeuchi $Y$, Chatani $R$ et al. Prospective evaluation of narrow-band imaging endoscopy for screening of esophageal squamous mucosal high-grade neoplasia in experienced and less experienced endoscopists. Dis Esophagus 2010; 23: 480-486

21 Lee CT, Chang CY, Lee YC et al. Narrow-band imaging with magnifying endoscopy for the screening of esophageal cancer in patients with primary head and neck cancers. Endoscopy 2010; 42: 613-619

22 Yoshimura N, Goda K, Tajiri H et al. Diagnostic utility of narrow-band imaging endoscopy for pharyngeal superficial carcinoma. World J Gastroenterol 2011; 17: 4999-5006 
23 Tanaka S, Morita Y, Fujita T et al. Clinicopathological characteristics of abnormal micro-lesions at the oro-hypopharynx detected by a magnifying narrow band imaging system. Dig Endosc 2012; 24: 100-109

24 Shimizu Y, Tsukagoshi H, Fujita $M$ et al. Head and neck cancer arising after endoscopic mucosal resection for squamous cell carcinoma of the esophagus. Endoscopy 2003; 35: 322-326

25 Katada C, Nakayama M, Tanabe $S$ et al. Narrow band imaging for detecting metachronous superficial oropharyngeal and hypopharyngeal squamous cell carcinoma after chemoradiotherapy for head and neck cancers. Laryngoscope 2008; 118: 1787-1790

26 Katada C, Tanabe S, Koizumi $W$ et al. Narrow band imaging for detecting superficial squamous cell carcinoma of the head and neck in patients with esophageal squamous cell carcinoma. Endoscopy 2010; 42: $185-190$

27 Shiozaki $H$, Tahara $H$, Kobayashi $K$ et al. Endoscopic screening of early esophageal cancer with the lugol dye method in patients with head and neck cancers. Cancer 1990; 66: 2068-2071
28 Ina H, Shibuya H, Ohashi I et al. The frequency of a concomitant early esophageal cancer in male patients with oral and oropharyngeal cancer. Cancer 1994; 73: 2038 -2041

29 Goda K, Dobashi A, Tajiri H. Perspectives on narrow-band imaging endoscopy for superficial squamous neoplasms of the orohypopharynx and esophagus. Dig Endosc 2014; 26: 1-11

30 Takahashi M, Shimizu Y, Ono M et al. Endoscopic diagnosis of early neoplasia of the esophagus with narrow band imaging: correlations among background coloration and iodine staining findings. J Gastroenterol Hepatol 2014; 29: $762-768$

31 Shimizu M, Nagata K, Yamaguchi H et al. Squamous intraepithelial neoplasia of the esophagus: past, present, and future. J Gastroenterol 2009; 44: 103 - 112

32 Fujii S, Yamazaki M, Muto M et al. Microvascular irregularities are associated with composition of squamous epithelial lesions and correlate with subepithelial invasion of superficial-type pharyngeal squamous cell carcinoma. Histopathology 2010; 56: 510-522 